\title{
Optical detection of paramagnetic centres: from crystals to glass-ceramics
}

\author{
Uldis Rogulis \\ Institute of Solid State Physics, University of Latvia, 8 Kengaraga Str., Riga LV-1063, Latvia \\ E-mail: rogulis@latnet.lv
}

Received January 18, 2016, published online May 25, 2016

\begin{abstract}
An unambiguous attribution of the absorption spectra to definite paramagnetic centres identified by the EPR techniques in the most cases is problematic. This problem may be solved by applying of a direct measurement techniques - the EPR detected via the magnetic circular dichroism, or briefly MCD-EPR. The present survey reports on the advantages and disadvantages applying the MCD-EPR techniques to simple and complex paramagnetic centres in crystals as well as glasses and glass-ceramics.

PACS: $76.30 \mathrm{Da}$ Ions and impurities: general;

$76.70 \mathrm{Hb}$ Optically detected magnetic resonance (ODMR).
\end{abstract}

Keywords: EPR, ODMR, magnetic circular dichroism, paramagnetic centres, point defects

\section{Introduction}

Magnetic resonance technique, particularly electron paramagnetic resonance (EPR) (or alternatively, electron spin resonance, ESR) spectroscopy is the powerful method for structural characterization of paramagnetic point defects in solids [1-10] which allows the determination (1) nature and valence of the impurity; (2) nature and number of ligands; (3) symmetry of the complex; (4) possible presence of nearby defects; (5) true metal-ligand distance and its dependence on pressure and temperature etc. The optically detected magnetic resonance (ODMR) allows the investigation of the structure of luminescence and colour centres [11].

In this survey we report on the advantages and disadvantages when applying the EPR detected via the magnetic circular dichroism (MCD-EPR) techniques to simple and complex paramagnetic centres in crystals as well as glasses and glass-ceramics. The MCD-EPR is one of the variations of the optically-detected magnetic resonance techniques [11].

The main advantages of the MCD-EPR are:

- the linkage between the paramagnetic and optical properties of the colour centre could be estimated directly;

- if several optical bands overlap, they could be separated;

- in several cases, it is possible to identify the structure of the paramagnetic (PM) centre by the MCD-EPR angular dependencies;
- after the magnetic circular dichroism is identified, it is possible to follow the changes of the defect concentration involved in definite processes via the MCD changes. It is especially useful, if the absorption bands overlap, but the MCD bands have very characteristic features.

There are some disadvantages and restrictions:

- the MCD-EPR linewidths is, as a rule, larger than the corresponding EPR lines, mainly due to the lower homogeneity of the ODMR magnets;

— not all the absorption bands possess an intense MCD signal;

- if the MCD-EPR is structureless or its angular dependencies are not pronounced, a further characterisation is possible only if the PM centre has a well parametrised EPR spectrum.

We will discuss the experimental aspects of the MCDEPR and then give several examples each characterising the information available, when applying the MCD-EPR to the optical detection of the paramagnetic centres in crystals, glasses and glass-ceramics.

\section{Experimental aspects}

The MCD-EPR spectrometer is, as a rule, custom-built low-temperature one. The details are described elsewhere [11]. It consists of the following main units: magnetooptical cryostat with a sample cavity, microwave accessories and a circular polarisation unit. While the EPR spectrometers usually work using the X-band microwaves, the MCD-EPR spectrometers are, as a rule, equipped with at 
least $24 \mathrm{GHz}$ microwave source, often also with $36 \mathrm{GHz}$ to $45 \mathrm{GHz}$ or even 72 or $95 \mathrm{GHz}$ sources. The higher microwave frequencies for the MCD-EPR techniques are necessary to reach higher magnetic fields for the MCD and a better resolution for the MCD-EPR spectra. The necessary magnetic fields are from at least $2 \mathrm{~T}$ for the $24 \mathrm{GHz}$ microwave band up to at least $4 \mathrm{~T}$ for the $93 \mathrm{GHz}$ microwave range. Therefore, the superconducting magnets at liquid helium temperatures should be used.

At first, the PM part of the MCD should be separated [11]. It could be reached by comparing the MCD at two different temperatures, for example, 4.2 and $1.5 \mathrm{~K}$. The PM MCD part at $1.5 \mathrm{~K}$ is about of 2 times stronger compared to the MCD at $4.2 \mathrm{~K}$.

The MCD-EPR measurement is performed by scanning the magnetic field at some of PM MCD wavelengths and applied microwave power [11].

So-called "tagged MCD" spectra [11] could be measured, by switching on-off microwave power at different MCD wavelengths.

The most complicated version of the techniques is the so-called optically-detected ENDOR [12], however, rarely applicable due to its technical complexity.

\section{Results and discussion}

To demonstrate the examples of the MCD-EPR application and the information available, we separated them in subsections 3.1.-3.4.

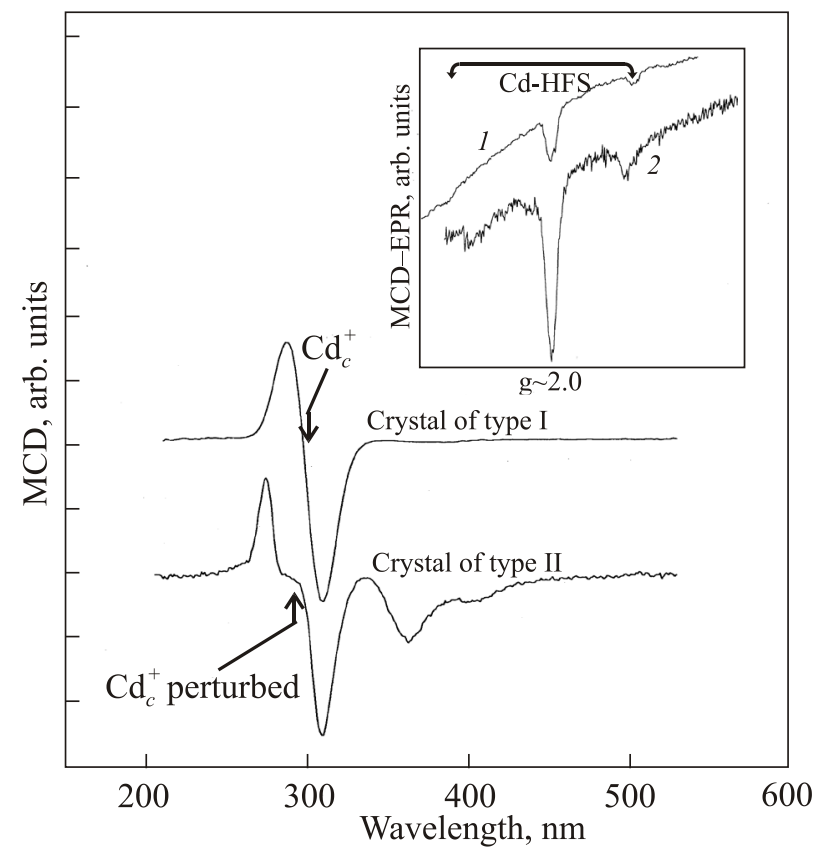

Fig. 1. $\mathrm{MCD}$ of the $\mathrm{BaF}_{2}$ crystals of two types measured at $B=2 \mathrm{~T}$; inset: MCD-EPR of the $\mathrm{Cd}_{c}^{+}$centre measured at $310 \mathrm{~nm} \mathrm{MCD}$ (curve 1) and of the $\mathrm{Cd}_{c}^{+}$perturbed centre measured at $300 \mathrm{~nm}$ MCD (curve 2) in the $53 \mathrm{GHz}$ microwave range. Cd hyperfine splitting for the perturbed centre is smaller as for the unperturbed centre.

\subsection{Estimation of the EPR parameters through the $M C D-E P R$}

At first, we discuss the possibilities to estimate some of the EPR parameters through the MCD-EPR by the examples of Cd-centres in $\mathrm{BaF}_{2}$.

Optical absorption and MCD spectra (shown in Fig. 1.) of $\gamma$-irradiated $\mathrm{BaF}_{2}$ single crystals of type I and II are different (see details in [13]).

To find out the nature of the centres responsible for these spectra, the MCD-EPR techniques has been applied. The MCD-EPR spectra shown in the inset of the Fig. 1, reveal two different hyperfine structure (HFS) lines in both types of $\mathrm{BaF}_{2}$. The first one belong to the $\mathrm{Cd}_{c}^{+}$centre and the second one, with slightly smaller HFS - to a perturbed Cd-related centre [13]. According to the EPR data, this centre has a superhyperfine (SHF) interaction with only 7 fluorine, i.e., one fluorine would be substituted by an ion without resolved SHF structure [14].

\subsection{Estimation of the optical bands of centres through the "tagged MCD”}

The second line of experimental possibilities of the MCD-EPR techniques is the estimation of the optical bands of centres through the "tagged MCD" on the example of $\mathrm{Ga}^{2+}$ hole centres in $\mathrm{RbBr}$.

Ga-related centres, especially hole centres, have been widely investigated. However, only absorption or even MCD measurements alone didn't allowed to identify different $\mathrm{Ga}^{2+}$ hole centres [15]. The MCD-EPR spectra shown in Fig. 2 (based on [15]) allowed estimate the HFS parameters of two different $\mathrm{Ga}^{2+}$ hole centres, which MCD spectra significantly differ.

These different MCD spectra could be the most clearly resolved by so-called "tagged MCD" techniques, switching on-off microwaves at a fixed resonance field and recording

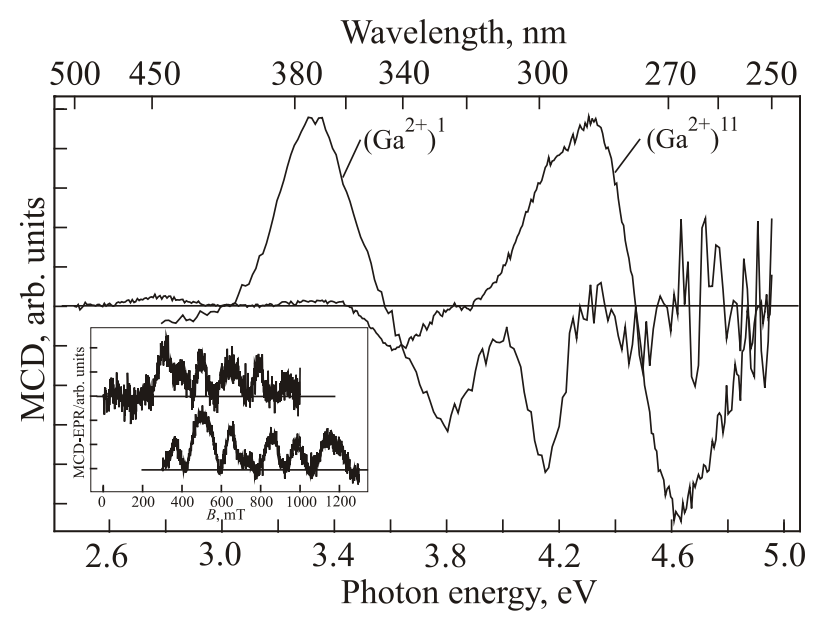

Fig. 2. "Tagged MCD" of two $\mathrm{Ga}^{2+}$ centres in an x-irradiated $\mathrm{RbBr}: \mathrm{Ga}$ crystal measured at $1.5 \mathrm{~K}$; inset: MCD-EPR of the $\left(\mathrm{Ga}^{2+}\right)$ ' centre measured at $370 \mathrm{~nm}$ (upper curve) and $\left(\mathrm{Ga}^{2+}\right)$ ", centre detected at $289 \mathrm{~nm}$ at the $23.9 \mathrm{GHz}$ microwave frequency. 


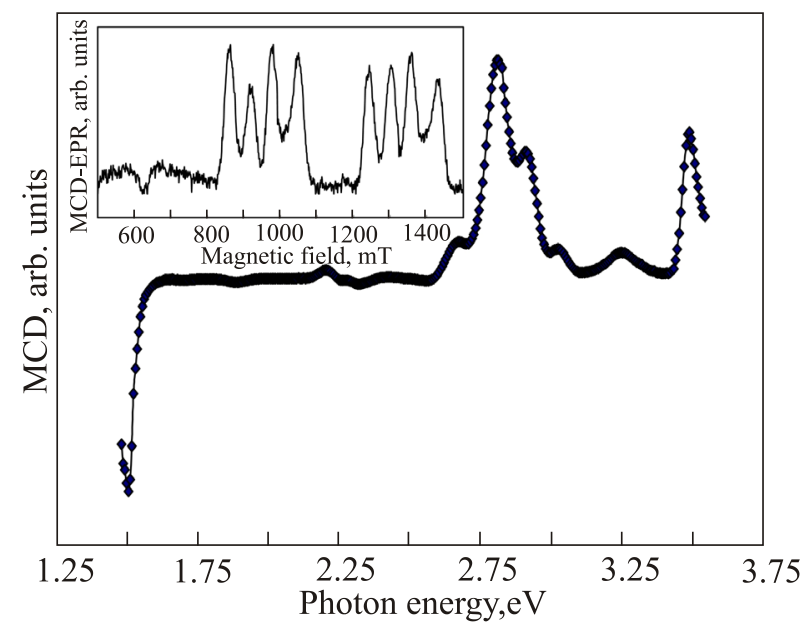

Fig. 3. MCD of a $\gamma$-irradiated at $295 \mathrm{~K} \mathrm{CsI:Tl} \mathrm{crystal} \mathrm{measured} \mathrm{at}$ $T=1.5 \mathrm{~K}$ and $B=2 \mathrm{~T}$; inset: MCD-EPR of the Tl-trimer centre measured at $425 \mathrm{~nm} \mathrm{MCD}$ and $24.32 \mathrm{GHz}$ microwave frequency at $\mathbf{B} \|[100]$.

the microwave-induced changes for all the MCD wavelengths. These knowledge of the MCD bands allowed to follow the recharging processes in the $\mathrm{x}$-irradiated $\mathrm{RbBr}: \mathrm{Ga}$ crystals [15]. Similar research was successful in CsBr:Ga and RbI:Tl crystals as well [16].

\subsection{Structural identification of centres through the $M C D-E P R(\mathrm{CsI}-\mathrm{Tl})$}

In CsI-Tl crystals, after $\mathrm{x}$ - or $\gamma$-irradiation, the induced absorption and MCD spectra have been obtained, however, the conventional EPR technique failed to give results. Therefore, the MCD-EPR technique is the only one allowing to get magnetic resonance spectra and to offer the possibility for the defect structure identification. The inset in Fig. 3 (see details in [17]) shows the MCD-EPR spectrum taken at the MCD-wavelength of $425 \mathrm{~nm}$ and $\mathbf{B} \|$ [100].

Analysing the angular dependencies of the spectrum, the model of the Tl-related centre consisting of three adjacent $\mathrm{Tl}$ ions has been estimated [17].

\subsection{Estimation of the optical transition range for the centre with known EPR}

The first example is concerned with the optical transition range of the $F$-type centres in $\mathrm{LiBaF}_{3}$ crystals.

EPR measurements on $\mathrm{LiBaF}_{3}$ crystals, $\mathrm{x}$-irradiated at room temperature (RT) and recombination luminescence detected EPR measurements on the samples $\mathrm{x}$-irradiated at 4.2 K (see details in [18]) showed the presence of several $F$-type centres, each with different EPR parameters. The examination of this question by the MCD and MCD-EPR techniques (Fig. 4), showed that the MCD bands created after $\mathrm{x}$-irradiation at RT and liquid-helium temperature are different as well.

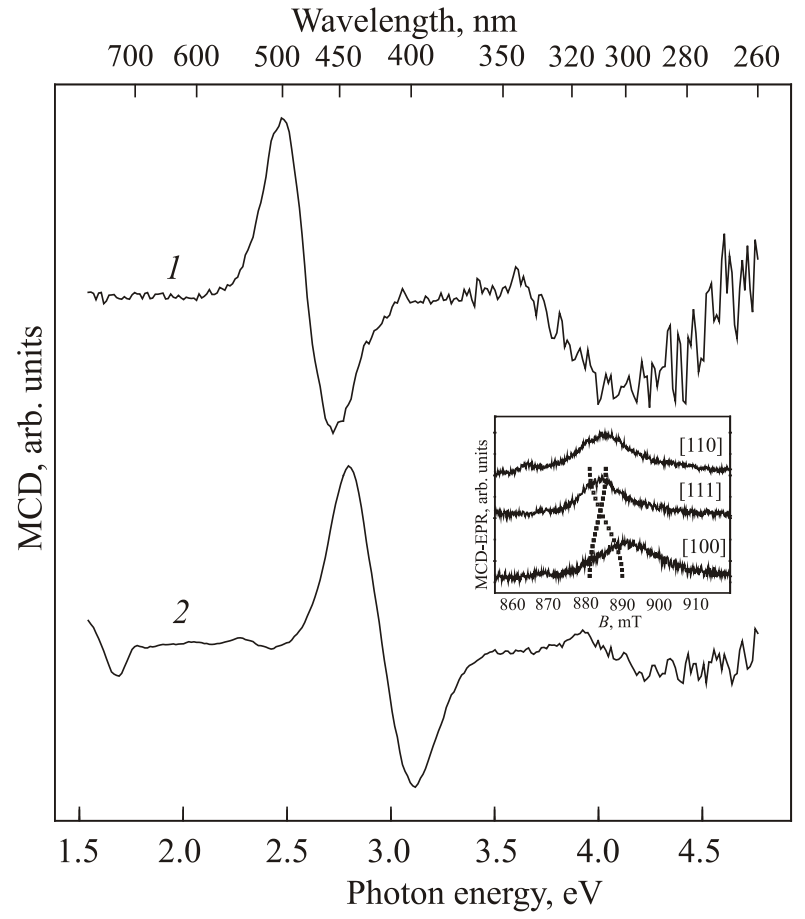

Fig. 4. $\mathrm{MCD}$ of a $\mathrm{LiBaF}_{3}$ crystal (1) after $\mathrm{x}$-irradiation at $4.2 \mathrm{~K}$ and (2) after x-irradiation at RT measured at $1.5 \mathrm{~K}$ and $B=1 \mathrm{~T}$; inset: MCD-EPR of the $F$-type centre in $\mathrm{LiBaF}_{3} \mathrm{x}$-irradiated at $4.2 \mathrm{~K}$, measured at $500 \mathrm{~nm} \mathrm{MCD}$ for different magnetic field orientations and microwave frequency of $24.42 \mathrm{GHz}$.

The MCD-EPR showed different broad resonance lines, while the characteristic symmetry of the $F$-type centres remains [18]. Therefore, the presence of these $F$-type centres in $\mathrm{LiBaF}_{3}$ has been stated and the corresponding MCD and absorption spectral regions have been estimated [18].

The second case is concerned with the optical transition range of the phosphate radical centres in phosphate glasses.

Phosphate glasses could be coloured by $\mathrm{x}$-irradiation. The creation of $\mathrm{PO}_{4}^{2-}$ and $\mathrm{PO}_{3}^{2-}$ radicals has been estimated by the EPR [19]. However, the correlation between PM radicals and induced absorption bands has not been estimated by direct methods. The MCD spectra shown in Fig. 5 (based on [20]), alone also do not allow to do conclusions, however, measuring the MCD-EPR (see inset), the observed HFS is the same as that estimated earlier by the EPR.

Therefore, it was concluded that both $\mathrm{PO}_{4}^{2-}$ and $\mathrm{PO}_{3}^{2-}$ radicals have very similar absorption shape and are indeed responsible for the colouring of the phosphate glasses by $\mathrm{x}$-rays.

The last example is concerned with the optical transition range of the $\mathrm{Gd}^{3+}$ centres in $\mathrm{CaF}_{2}$ crystallites in the oxyfluoride glass-ceramics.

The analysis of the nature of absorption bands of impurity/defect centres is complicated, especially if these bands are broad. It is even more difficult to decide which of the bands belong to the glasses and which to the crystallites in the glass-ceramics. As we observed by the example of $\mathrm{Gd}^{3+}$-doped oxyfluorides, the MCD spectra of the glass 


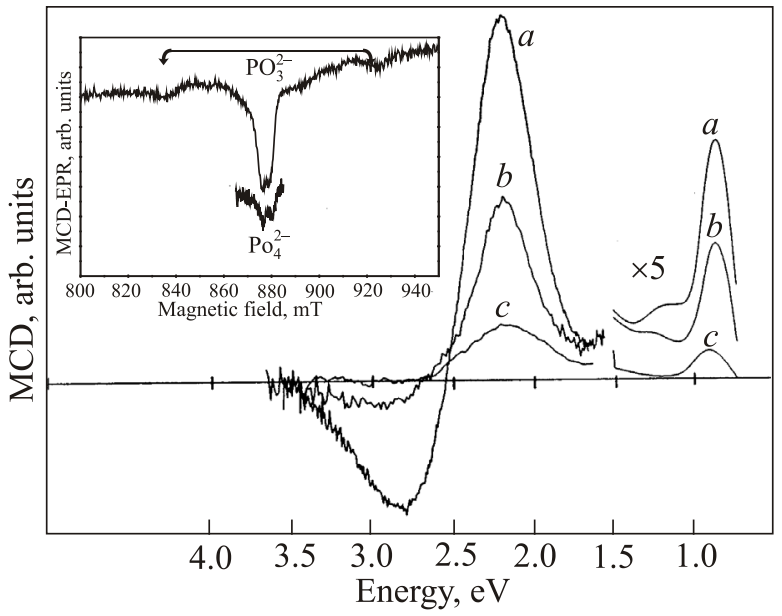

Fig. 5. $\mathrm{MCD}$ of $\mathrm{CaO}-\mathrm{P}_{2} \mathrm{O}_{5}$ glasses $\mathrm{x}$-irradiated at $\mathrm{RT}(a)$ and "tagged MCD" spectra measured for $\mathrm{PO}_{4}^{2-}$ centre $(b)$ and $\mathrm{PO}_{3}^{2-}$ centre $(c)$; inset: MCD-EPR of the irradiated glass measured at $2.2 \mathrm{eV} \mathrm{MCD}$ for $1.5 \mathrm{~K}$ (upper curve) and $4.2 \mathrm{~K}$ (lower curve) at the $24.1 \mathrm{GHz}$ microwave frequency.

and glass-ceramics seemed to be very similar. The solution has again been found by the MCD-EPR techniques. In glasses, no MCD-EPR signal could be observed. The EPR spectra of the $\mathrm{Gd}^{3+}$ centres in glasses are typical of lowsymmetry centres and the EPR is therefore broadened over a whole magnetic field range. On the contrary, the EPR of glass-ceramics showed spectra of the cubic $\mathrm{Gd}^{3+}$ centres with well resolved fine structure [21]. The same $\mathrm{Gd}^{3+}$ spectrum but without so good resolved fine structure has been detected also in the oxyfluoride glass-ceramics (see Fig. 6, based on [21]).

Therefore, the MCD-EPR technique allows to find out a direct correlation between the cubic $\mathrm{Gd}^{3+}$ centre and its MCD, but a similar MCD in the glasses could be attributed to the low-symmetry $\mathrm{Gd}^{3+}$ centres [21].

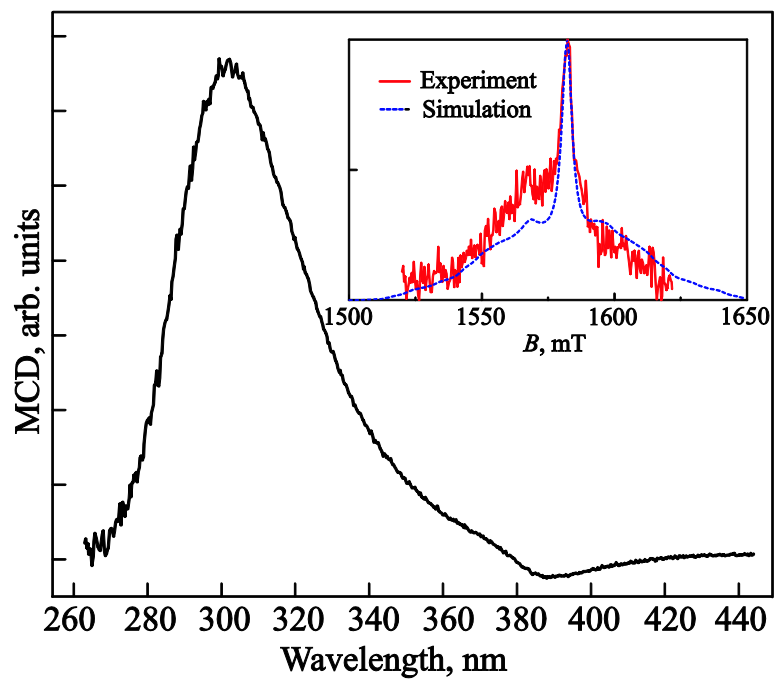

Fig. 6. MCD of an oxyfluoride glass-ceramic sample measured at $4.2 \mathrm{~K}$ and $B=1 \mathrm{~T}$; inset: the experimental MCD-EPR spectrum and simulated spectrum with the EPR parameters for a cubic $\mathrm{Gd}^{3+}$ centre in a $\mathrm{CaF}_{2}$ crystal.

\section{Conclusions}

It is shown how the MCD-EPR techniques could be successfully applied for optical characterization and identification of many paramagnetic centres in crystals, glasses and glass-ceramics.

The main advantages are: the possibility of direct attribution of the optical (MCD) transitions to certain paramagnetic centres; the possibility to resolve the overlapped absorption (MCD) bands of several centres and to follow the behaviour of the PM centres during the processes; the possibility to identify the structure of a PM centre if its identifying via EPR is not possible.

The following disadvantages should be taken into account: the lower resolution of the MCD-EPR spectra; nonavailability of the measurements if the MCD is very weak or absent at all; difficulties to identify the MCD-EPR spectra if they are poorly resolved and the EPR data are principally not available.

Nevertheless, the MCD-EPR with described above restrictions has positioned itself as a powerful tool for direct identification of the optical transition range of paramagnetic centres. Its application field includes structural identification of complex paramagnetic centres in crystals as well as amorphous structures-glasses and glass-ceramics.

This work was supported by Latvian Science Council project No 302/2012.

1. J.M. Spaeth and F. Lohse, J. Phys. Chem Solids 51, 861 (1990).

2. M. Moreno, J. Phys. Chem Solids 51, 835 (1990).

3. Y. Chen and M.M. Abraham, J. Phys. Chem Solids 51, 747 (1990).

4. J.M. Spaeth, Radiation Measurements 33, 527 (2001).

5. A.C Lushchik and A.G. Frorip, Phys. Status Solidi B 161, 525 (1990).

6. P. Fattibene and F. Callens, Appl. Radiation and Isotopes $\mathbf{6 8}$, 2033 (2010).

7. U. Eichhoff and P. Hoefer, Fiz. Nizk. Temp. 41, 81 (2015) [Low Temp. Phys. 41, 62 (2015)].

8. Yu.Yu. Titova, L.B. Belykh, and F.K. Schmidt, Fiz. Nizk. Temp. 41, 38 (2015) [Low Temp. Phys. 41, 25 (2015)].

9. Z.H. Zhang, S.Y. Wu, M.Q. Kuang, and C.C. Dong, Fiz. Nizk. Temp. 41, 223 (2015) [Low Temp. Phys. 41, 177 (2015)].

10. V.A. Shapovalov, E.S. Zhitlukhina, K.V. Lamonova, S.M. Orel, S.N. Barilo, and Yu.G. Pashkevich, Fiz. Nizk. Temp. 40, 595 (2014) [Low Temp. Phys. 40, 462 (2014)].

11. J.-M. Spaeth and H. Overhof, Point Defects in Semiconductors and Insulators, Springer-Verlag, Berlin, Heidelberg (2003).

12. M. Fockele, F. Lohse, J.-M. Spaeth, and R.H. Bartram, J. Phys.: Condens. Matter 1, 13 (1989).

13. U. Rogulis, J. Trokss, A. Veispals, I. Tale, P. Kulis, and M. Springis, Rad. Effects and Defects in Solids 135, 361 (1995).

14. A. Fedotovs and U. Rogulis, Latvian J. Phys. and Techn. Sci. 3, 62 (2005). 
15. U. Rogulis, S. Schweizer, S. Assmann, and J.-M. Spaeth, J. Appl. Phys. 84, 4537 (1998).

16. U. Rogulis, C. Dietze, Th. Pawlik, Th. Hangleiter, and J.-M. Spaeth, J. Appl. Phys. 80, 2430 (1996).

17. U. Rogulis, J.-M. Spaeth, E. Elsts, and A. Dolgopolova, Rad. Measurements 38, 389 (2004).

18. U. Rogulis, J.-M. Spaeth, I. Tale, M. Nik1, N. Ichinose, and K. Shimamura, Rad. Measurements 38, 663 (2004).
19. T.V. Bocharova, G.O. Karapetyan, and O.A. Yaschurszhinskaya, Sov. J Glass Phys. and Chem. 11, 409 (1985).

20. D. Brics, J. Ozols, U. Rogulis, J. Trokss, W. Meise, and J.-M. Spaeth, Solid State Commun. 81, 745 (1992).

21. A. Fedotovs, A. Antuzevics, U. Rogulis, M. Kemere, and R. Ignatans, J. Non-Crystalline Solids 429, 118 (2015). 